Research Article

\title{
Schur $m$-Power Convexity of a Class of Multiplicatively Convex Functions and Applications
}

\author{
Wen Wang ${ }^{1}$ and Shiguo Yang ${ }^{1,2}$ \\ ${ }^{1}$ School of Mathematics and Statistics, Hefei Normal University, Hefei 230601, China \\ ${ }^{2}$ Department of Mathematics and Physics, Anhui Xinhua University, Hefei 230088, China
}

Correspondence should be addressed to Wen Wang; wenwang1985@163.com

Received 14 January 2014; Revised 2 April 2014; Accepted 23 April 2014; Published 20 May 2014

Academic Editor: Marco Sabatini

Copyright (c) 2014 W. Wang and S. Yang. This is an open access article distributed under the Creative Commons Attribution License, which permits unrestricted use, distribution, and reproduction in any medium, provided the original work is properly cited.

We investigate the conditions under which the symmetric functions $F_{n, k}(\mathbf{x}, r)=\prod_{1 \leq i_{1}<i_{2}<\cdots<i_{k} \leq n} f\left(\left(\sum_{j=1}^{k} x_{i_{j}}^{r}\right)^{1 / r}\right), k=1,2, \ldots, n$, are Schur $m$-power convex for $x \in \mathbb{R}_{++}^{n}$ and $r>0$. As a consequence, we prove that these functions are Schur geometrically convex and Schur harmonically convex, which generalizes some known results. By applying the theory of majorization, several inequalities involving the $p$ th power mean and the arithmetic, the geometric, or the harmonic means are presented.

\section{Introduction}

Let $\mathbb{R}^{n}=\left\{\left(x_{1}, x_{2}, \ldots, x_{n}\right): x_{i} \in \mathbb{R}, i=1,2, \ldots, n\right\}$ and $\mathbb{R}_{++}^{n}=$ $\left\{\left(x_{1}, x_{2}, \ldots, x_{n}\right): x_{i}>0, i=1,2, \ldots, n\right\}$. In particular, $\mathbb{R}_{++}=$ $(0, \infty)$. For $\mathbf{x}=\left(x_{1}, x_{2}, \ldots, x_{n}\right)$, we denote by

$$
\begin{gathered}
\mathbf{x}=\left(x_{1}, x_{2}, \ldots, x_{n}\right), \\
\ln (\mathbf{x})=\left(\ln x_{1}, \ln x_{2}, \ldots, \ln x_{n}\right), \\
\frac{1}{\mathbf{x}}=\left(\frac{1}{x_{1}}, \frac{1}{x_{2}}, \ldots, \frac{1}{x_{n}}\right) .
\end{gathered}
$$

The Hamy symmetric function $[1,2]$ is defined as

$$
\begin{array}{r}
F_{n}(\mathbf{x}, r)=F_{n}\left(x_{1}, x_{2}, \ldots, x_{n} ; r\right)=\sum_{1 \leq i_{1}<i_{2}<\cdots<i_{r} \leq n}\left(\prod_{j=1}^{r} x_{i_{j}}\right)^{1 / r}, \\
r=1,2, \ldots, n .
\end{array}
$$

The properties and applications of Hamy symmetric function can be found in the book of Bullen et al. [1].

Throughout the paper, let $I \subset \mathbb{R}_{++}$and $I^{n} \subset \mathbb{R}_{++}^{n}$.
In 2007, Guan [3] defined a more general symmetric function: let $f: I^{n} \rightarrow \mathbb{R}_{++}$, and

$$
\begin{array}{r}
\sum_{n}^{r}(f(x))=\sum_{1 \leq i_{1}<i_{2}<\cdots<i_{r} \leq n} f\left(\prod_{j=1}^{r} x_{i_{j}}^{1 / r}\right), \\
r=1,2, \ldots, n,
\end{array}
$$

where $i_{1}, i_{2}, \ldots, i_{n}$ are nonnegative integers, $r \in N=\{1,2$, $3, \ldots\}$. Guan proved that the above symmetric function is Schur geometrically convex on $I^{n}$.

In 2010, Rovența [4] defined the following symmetric function. Let $f: I \rightarrow \mathbb{R}_{++}$be a log-convex function

$$
\begin{array}{r}
F_{k}(\mathbf{x})=\sum_{1 \leq i_{1}<i_{2}<\cdots<i_{k} \leq n} \prod_{j=1}^{k} f\left(x_{i_{j}}\right), \\
k=1,2, \ldots, n .
\end{array}
$$

Rovența proved that (4) is a Schur convex function on $I^{n}$. 
In 2010, Meng et al. [5] proved the dual form of the Hamy symmetric function

$$
\begin{array}{r}
H_{n}(\mathbf{x}, r)=\prod_{1 \leq i_{1}<i_{2}<\cdots<i_{k} \leq n}\left(\sum_{j=1}^{r} x_{i_{j}}^{1 / r}\right), \\
k=1,2, \ldots, n,
\end{array}
$$

was Schur harmonically convex in $\mathbb{R}_{++}^{n}$.

In 2013, Shi and Zhang [6] investigated the following dual form of $F_{k}(\mathbf{x})$ :

$$
F_{k}^{*}(\mathbf{x})=\prod_{1 \leq i_{1}<i_{2}<\cdots<i_{k} \leq n} \sum_{j=1}^{k} f\left(x_{i_{j}}\right), \quad k=1,2, \ldots, n .
$$

They proved that $F_{k}^{*}(\mathbf{x})$ is Schur convex, Schur geometrically and harmonically convex on $I^{n}$.

Recently, Yang [7-9] generalized the notion of Schur convexity to Schur $m$-power convexity, which contains the Schur convexity, Schur geometrical convexity, and Schur harmonic convexity. Moreover, he discussed Schur $m$-power convexity of Stolarsky means [7], Gini means [8], and Daróczy means [9]. Wang and Yang showed that generalized Hamy symmetric function [10] and a class of symmetric functions [11] are Schur $m$-power convex.

Now we define the more general dual form of symmetric function.

Definition 1. Let $\Omega \subset \mathbb{R}_{++}^{n}$ be a symmetric convex set with nonempty interior and $f: \Omega \rightarrow \mathbb{R}_{++}$is continuous on $\Omega$ and differentiable in the interior of $\Omega$. For $r>0$, define the symmetric functions $F_{n, k}(\mathbf{x}, r)$ by

$$
\begin{array}{r}
F_{n, k}(\mathbf{x}, r)=\prod_{1 \leq i_{1}<i_{2}<\cdots<i_{k} \leq n} f\left(\left(\sum_{j=1}^{k} x_{i_{j}}^{r}\right)^{1 / r}\right), \\
k=1,2, \ldots, n .
\end{array}
$$

In this paper, we investigate the Schur $m$-power convexity of the above more general dual form of symmetric functions. In particular, we obtain that the above more general dual form of symmetric functions is Schur geometrically convex and Schur harmonically convex, which generalizes some known results. As a consequence, we are able to prove a number of new inequalities concerning the $p$ th power mean, the arithmetic mean, and the geometric and the harmonic mean.

\section{Definitions and Lemmas}

We first recall several definitions as follows.

Definition 2 (see $[12,13])$. Let $\mathbf{x}=\left(x_{1}, x_{2}, \ldots, x_{n}\right)$ and $\mathbf{y}=$ $\left(y_{1}, y_{2}, \ldots, y_{n}\right) \in \mathbb{R}^{n}$.

(1) $\mathbf{x} \geq \mathbf{y}$ means $x_{i} \geq y_{i}$ for all $i=1,2, \ldots, n$.

(2) Let $\Omega \subset \mathbb{R}^{n}, f: \Omega \rightarrow \mathbb{R}$ is said to be increasing if $\mathbf{x} \geq \mathbf{y}$ implies $f(\mathbf{x}) \geq f(\mathbf{y})$. $f$ is said to be decreasing if and only if $-f$ is increasing.
Definition 3 (see $[12,13])$. Suppose that $\mathbf{x}=\left(x_{1}, x_{2}, \ldots, x_{n}\right)$ and $\mathbf{y}=\left(y_{1}, y_{2}, \ldots, y_{n}\right) \in \mathbb{R}^{n}$ are two $n$-tuples real numbers.

(1) $\mathbf{y}$ majorizes $\mathbf{x}$ (in symbols $\mathbf{x} \prec \mathbf{y}$ ), if $\sum_{i=1}^{k} x_{[i]} \leq$ $\sum_{i=1}^{k} y_{[i]},(k=1,2, \ldots, n-1)$ and $\sum_{i=1}^{n} x_{[i]}=\sum_{i=1}^{n} y_{[i]}$, where $x_{[1]} \geq x_{[2]} \geq \cdots \geq x_{[n]}, y_{[1]} \geq y_{[2]} \geq \cdots \geq y_{[n]}$ are rearrangements of $\mathbf{x}$ and $\mathbf{y}$ in a descending order.

(2) A real-valued function $f: \Omega \subset \mathbb{R}^{n} \rightarrow \mathbb{R}$ is said to be Schur convex on $\Omega$ if

$$
\mathbf{x} \prec \mathbf{y} \quad \text { on } \Omega \Longrightarrow f(\mathbf{x}) \leq f(\mathbf{y}) .
$$

$f$ is a Schur concave function on $\Omega$ if and only if $-f$ is a Schur convex function.

Definition 4 (see [14]). Suppose $\mathbf{x}=\left(x_{1}, x_{2}, \ldots, x_{n}\right)$ and $\mathbf{y}=$ $\left(y_{1}, y_{2}, \ldots, y_{n}\right) \in \mathbb{R}^{n}$ are two $n$-tuples real numbers. Let $\Omega \subset$ $\mathbb{R}_{++}^{n}$. A function $f: \Omega \rightarrow \mathbb{R}_{++}$is called Schur geometrically convex if

$$
\ln \mathbf{x} \prec \ln \mathbf{y} \quad \text { on } \Omega \Longrightarrow \varphi(\mathbf{x}) \leq \varphi(\mathbf{y}) .
$$

$f$ is Schur geometrically concave if $-f$ is Schur geometrically convex.

The following Theorem is basic and plays an important role in the theory of the Schur geometrically convex function.

Lemma 5 (see [14]). Let $\varphi(\mathbf{x})=\varphi\left(x_{1}, x_{2}, \ldots, x_{n}\right)$ be symmetric and continuous on $\Omega \subset \mathbb{R}_{++}^{n}$ and differentiable in $\Omega^{0}$. Then $\varphi: \Omega \rightarrow \mathbb{R}_{++}$is Schur geometrically convex (Schur geometrically concave) if and only if

$$
\left(\ln x_{1}-\ln x_{2}\right)\left(x_{1} \frac{\partial \varphi}{\partial x_{1}}-x_{2} \frac{\partial \varphi}{\partial x_{2}}\right) \geq 0(\leq 0) .
$$

Definition 6 (see $[15,16])$. Let $\Omega \in \mathbb{R}^{n}$.

(1) $A$ set $\Omega$ is called harmonically convex if $\mathbf{x y} /(\lambda \mathbf{x}+(1-$ $\lambda \mathbf{y})) \in \Omega$ for every $\mathbf{x}, \mathbf{y} \in \Omega$ and $\lambda \in[0,1]$, where $\mathbf{x y}=\sum_{i=1}^{n} x_{i} y_{i}$ and $1 / \mathbf{x}=\left(1 / x_{1}, \ldots, 1 / x_{n}\right)$.

(2) A function $\varphi: \Omega \rightarrow \mathbb{R}_{++}$is called Schur harmonically convex on $\Omega$ if $1 / \mathbf{x} \prec 1 / \mathbf{y}$ implies $\varphi(\mathbf{x}) \leq$ $\varphi(\mathbf{y}) . f$ is Schur harmonically concave if $-f$ is Schur harmonically convex.

Lemma 7 (see $[15,16]$ ). Let $\Omega \in \mathbb{R}_{++}^{n}$ be a symmetric and harmonically convex set with inner points and let $\varphi$ : $\Omega \rightarrow \mathbb{R}_{++}$be a continuously symmetric function which is differentiable in $\Omega^{0}$. Then $\varphi$ is Schur harmonically convex (Schur harmonically concave) on $\Omega$ if and only if

$$
\left(x_{1}-x_{2}\right)\left(x_{1}^{2} \frac{\partial \varphi}{\partial x_{1}}-x_{2}^{2} \frac{\partial \varphi}{\partial x_{2}}\right) \geq 0(\leq 0) .
$$

Schur convex, Schur geometrically convex, and Schur harmonically convex were introduced by Marshall et al. [13], Zhang [14], and Chu and Sun [15], respectively, and played a key role in analytic inequalities [1-36]. Moreover, the theory of convex functions and Schur convex functions is one of the most important research fields in modern analysis and geometry. 

follows.

Recently, Yang presents the Schur $f$-convexity in [7] as

Definition 8 (see [7-9]). Let $\Omega \subseteq \mathbb{R}^{n}$ be a set with nonempty interior and $f$ a strictly monotone function defined on $\Omega$. Let

$$
\begin{aligned}
& f(\mathbf{x})=\left(f\left(x_{1}\right), f\left(x_{2}\right), \ldots, f\left(x_{n}\right)\right), \\
& f(\mathbf{y})=\left(f\left(y_{1}\right), f\left(y_{2}\right), \ldots, f\left(y_{n}\right)\right) .
\end{aligned}
$$

Then function $\varphi: \Omega \rightarrow \mathbb{R}$ is said to be Schur $f$-convex on $\Omega$ if $f(\mathbf{x}) \prec f(\mathbf{y})$ on $\Omega$ implies $\psi(\mathbf{x}) \leq \psi(\mathbf{y})$.

$\psi$ is said to be $\operatorname{Schur} f$-concave if $-\psi$ is $S \operatorname{schur} f$-convex.

Take $f(x)=x, \ln x, x^{-1}$ in Definition 8 , it yields the Schur convexity, Schur geometrical convexity, and Schur harmonic convexity. It is clear that the Schur $f$-convexity is a generalization of the Schur convexity mentioned above. In general, we have the following.

Definition 9 (see [7-9]). Let $f: \mathbb{R}_{++} \rightarrow \mathbb{R}$ be defined by $f(x)=\left(x^{m}-1\right) / m$ if $m \neq 0$ and $f(x)=\ln x$ if $m=0$. Then function $\psi: \Omega \subseteq \mathbb{R}_{++}^{n} \rightarrow \mathbb{R}$ is said to be Schur $m$-power convex on $\Omega$ if $f(\mathbf{x}) \prec f(\mathbf{y})$ on $\Omega$ implies $\psi(\mathbf{x}) \leq \psi(\mathbf{y})$.

$\psi$ is said to be Schur $m$-power concave if $-\psi$ is Schur $m$ power convex.

Lemma 10 (see [7-9]). Let $\psi: \Omega \subseteq \mathbb{R}_{++}^{n} \rightarrow \mathbb{R}$ be continuous on $\Omega$ and differentiable in $\Omega^{0}$. Then $\psi$ is schurm-power convex (Schur m-power concave) on $\Omega$ if and only if $\psi$ is symmetric on $\Omega$ and

$$
\begin{array}{r}
\frac{x_{1}^{m}-x_{2}^{m}}{m}\left(x_{1}^{1-m} \frac{\partial \varphi}{\partial x_{1}}-x_{2}^{1-m} \frac{\partial \varphi}{\partial x_{2}}\right) \\
\geq 0(\leq 0), \quad \text { if } m \neq 0, \\
\left(\ln x_{1}-\ln x_{2}\right)\left(x_{1} \frac{\partial \varphi}{\partial x_{1}}-x_{2} \frac{\partial \varphi}{\partial x_{2}}\right) \\
\geq 0(\leq 0), \quad \text { if } m \neq 0,
\end{array}
$$

hold for any $\mathbf{x}=\left(x_{1}, x_{2}, \ldots, x_{n}\right) \in \Omega^{0}$ with $x_{1} \neq x_{2}$, where $\Omega \subseteq \mathbb{R}_{++}^{n}$ is a symmetric set with nonempty interior $\Omega^{0}$.

The following lemma is clearly due to the monotonicity property of the function $x^{p}$ on $\mathbb{R}_{++}$.

Lemma 11 (see [10]). For $x_{1}, x_{2}>0$ with $x_{1} \neq x_{2}$, let $U$ be defined by

$$
U\left(p ; x_{1}, x_{2}\right):= \begin{cases}\frac{x_{1}^{p}-x_{2}^{p}}{p\left(x_{1}-x_{2}\right)}, & \text { if } p \neq 0, \\ \frac{\ln x_{1}-\ln x_{2}}{x_{1}-x_{2}}, & \text { if } p=0 .\end{cases}
$$

0.)

Then $\operatorname{sgn}\left(\left(x_{1}^{p}-x_{2}^{p}\right) / p\left(x_{1}-x_{2}\right)\right)=1$, (i.e., $U\left(p ; x_{1}, x_{2}\right)>$
Remark 12 (see [10]). By Lemma 11, we see that

$$
\begin{aligned}
\operatorname{sgn}\left(\frac{x_{1}^{p}-x_{2}^{p}}{p}\right) & =\operatorname{sgn}\left(x_{1}-x_{2}\right) \quad \text { if } p \neq 0, \\
\operatorname{sgn}\left(\ln x_{1}-\ln x_{2}\right) & =\operatorname{sgn}\left(x_{1}-x_{2}\right) .
\end{aligned}
$$

Then the two discrimination inequalities in Lemma 10 are equivalent to

$$
\left(x_{1}-x_{2}\right)\left(x_{1}^{1-m} \frac{\partial \varphi}{\partial x_{1}}-x_{2}^{1-m} \frac{\partial \varphi}{\partial x_{2}}\right) \geq(\leq) 0 .
$$

Definition 13 (see $[3,17]$ ). Function $f: I \subset R_{++} \rightarrow R_{++}$is said to be multipicatively convex if

$$
x, y \in I, \quad \mu \in[0,1] \Longrightarrow f\left(x^{1-\mu} y^{\mu}\right) \leq f(x)^{1-\mu} f(y)^{\mu} .
$$

The following results have been proven, respectively.

Lemma 14 (see [17]). A continuous function $f: I \subset R_{++} \rightarrow$ $R_{++}$is multiplicatively convex if and only if

$$
x, y \in I \Longrightarrow f(\sqrt{x y}) \leq \sqrt{f(x) f(y)},
$$

or

$$
x_{1}, \ldots, x_{n} \in I \Longrightarrow f\left(\sqrt[n]{x_{1}, \ldots, x_{n}}\right) \leq \sqrt[n]{f\left(x_{1}\right) \cdots f\left(x_{n}\right)} .
$$

Lemma 15 (see [17]). Assume that $f: I \subset R_{++} \rightarrow R_{++}$ is a differential function. Then the following assertions are equivalent.

(i) fis multiplicatively convex.

(ii) The function $x f^{\prime}(x) / f(x)$ is nondecreasing.

Moreover, if $f$ is twice differentiable, then $f$ is multiplicatively convex if and only if

$$
x\left[f(x) f^{\prime \prime}(x)-f^{\prime 2}(x)\right]+f(x) f^{\prime}(x) \geq 0 \quad \forall x>0 .
$$

\section{Main Results and Proof}

Our main results are stated as follows.

Theorem 16. If $f$ is increasing and multiplicatively convex, then for $m \leq 0$ and $r>0, F_{n, k}(\mathbf{x}, r)$ defined in (7) are Schur $m$-power convex on $\Omega$, where $k=1,2, \ldots, n$.

Take $m=0,-1$ in Theorem 16 , we get the following Corollaries.

Corollary 17. If $f$ is increasing and multiplicatively convex, then for any $k=1,2, \ldots, n$ and $r>0, F_{n, k}(\mathbf{x}, r)$ defined in (7) are Schur geometrically convex on $\Omega$. 
Corollary 18. If $f$ is increasing and multiplicatively convex, then for any $k=1,2, \ldots, n$ and $r>0, F_{n, k}(\mathbf{x}, r)$ defined in (7) are Schur harmonically convex function on $\Omega$.

Theorem 19. Let $\mathbf{x}=\left(x_{1}, x_{2}, \ldots, x_{n}\right) \in \mathbb{R}_{++}^{n}$, and $\sum_{i=1}^{n} x_{i}=s$. If $f$ is increasing and multiplicatively convex, then for any $k=$ $1,2, \ldots, n$ and $r>0$, one has

$$
\begin{array}{ll}
F_{n, k}\left(\frac{n-\mu}{s-\mu \mathbf{x}}, r\right) \leq F_{n, k}\left(\frac{1}{\mathbf{x}}, r\right), & 0 \leq \mu \leq 1 ; \\
F_{n, k}\left(\frac{n+\mu}{s+\mu \mathbf{x}}, r\right) \leq F_{n, k}\left(\frac{1}{\mathbf{x}}, r\right), & 0 \leq \mu \leq 1 .
\end{array}
$$

To prove the main results, we first establish some lemmas.

Lemma 20. Let the function $f: I \subset \mathbb{R}_{++} \rightarrow \mathbb{R}_{++}$be continuous on $\Omega$ and differentiable in the interior of $\Omega$. For $m \leq 0$, if $f$ is increasing and multiplicatively convex, then $x^{1-m} f^{\prime}(x) / f(x)$ is increasing.

Proof. Since $f$ is multiplicatively convex, and by using Lemma 15, we can easily see that $x f^{\prime}(x) / f(x)$ is increasing. Further, by applying $f(x) \geq 0$ and the monotonicity of $f$, it follows that $x^{1-m} f^{\prime}(x) / f(x)$ is also increasing for $x>0$ and $m \leq 0$.

Lemma 21. If $r>0, c>0$ and $m \leq 0$, the function $g(x)=$ $\left(x^{r}-c\right)^{(r-m) / r} / x^{r}$ is increasing.

Proof. We can easily derive that

$$
\begin{aligned}
g^{\prime}(x)=\frac{1}{x^{2 r}} & \frac{r-m}{r}\left(x^{r}-c\right)^{-m / r} \\
& \left.\cdot r x^{r-1} \cdot x^{r}-r x^{r-1}\left(x^{r}-c\right)^{(r-m) / r}\right] \\
= & \frac{1}{x^{2 r}} r x^{r-1}\left(x^{r}-c\right)^{-m / r}\left[-\frac{m}{r} x^{r}+c\right] \geq 0 .
\end{aligned}
$$

So the function $g(x)$ is increasing.

Lemma 22. Let $f: \Omega \subset \mathbb{R}_{++}^{n} \rightarrow \mathbb{R}_{++}$be continuous on $\Omega$ and differentiable in the interior of $\Omega$. For $m \leq 0$ and $r>0$, if $f$ is increasing and multiplicatively convex, then

$$
\begin{aligned}
I= & F_{n, 2}(\mathbf{x}, r)\left(x_{1}-x_{2}\right)\left(x_{1}^{r-m}-x_{2}^{r-m}\right) \\
& \times \frac{f^{\prime}\left[\left(x_{1}^{r}+x_{2}^{r}\right)^{1 / r}\right]}{f\left[\left(x_{1}^{r}+x_{2}^{r}\right)^{1 / r}\right]}\left(x_{1}^{r}+x_{2}^{r}\right)^{1 / r-1} \geq 0, \\
I I= & F_{n, 2}(\mathbf{x}, r)\left(x_{1}-x_{2}\right) \\
& \times \sum_{j=3}^{n}\left\{\frac{f^{\prime}\left[\left(x_{1}^{r}+x_{j}^{r}\right)^{1 / r}\right]}{f\left[\left(x_{1}^{r}+x_{j}^{r}\right)^{1 / r}\right]} \cdot\left(x_{1}^{r}+x_{j}^{r}\right)^{1 / r-1} \cdot x_{1}^{r-m}\right.
\end{aligned}
$$

$$
\begin{gathered}
-\frac{f^{\prime}\left[\left(x_{2}^{r}+x_{j}^{r}\right)^{1 / r}\right]}{f\left[\left(x_{2}^{r}+x_{j}^{r}\right)^{1 / r}\right]} \\
\left.\cdot\left(x_{2}^{r-m}+x_{j}^{r}\right)^{1 / r-1} \cdot x_{2}^{r}\right\} \geq 0 .
\end{gathered}
$$

Proof. Firstly, we prove that (23) holds. Since $m \leq 0$ and $r>0$, then

$$
\left(x_{1}-x_{2}\right)\left(x_{1}^{r-m}-x_{2}^{r-m}\right) \geq 0 \text {. }
$$

So, we deduce that $I \geq 0$.

Secondly, we prove that (24) holds. Set $u=\left(x_{1}^{r}+x_{j}^{r}\right)^{1 / r}$, $v=\left(x_{2}^{r}+x_{j}^{r}\right)^{1 / r}$, obviously, $u, v \in \Omega$. One can easily find that

$$
\begin{aligned}
I I= & F_{n, 2}(\mathbf{x}, r) \frac{x_{1}-x_{2}}{u-v}(u-v) \\
& \times \sum_{j=3}^{n}\left\{\frac{u f^{\prime}(u)}{f(u)} \cdot \frac{\left(u^{r}-x_{j}^{r}\right)^{(r-m) / r}}{u^{r}}\right. \\
& \left.-\frac{v f^{\prime}(v)}{f(v)} \cdot \frac{\left(u^{r}-x_{j}^{r}\right)^{(r-m) / r}}{v^{r}}\right\} .
\end{aligned}
$$

Because $f$ is multiplicatively convex, and by Lemma 15 , we get

$$
(u-v)\left(\frac{u f^{\prime}(u)}{f(u)}-\frac{v f^{\prime}(v)}{f(v)}\right) \geq 0 .
$$

On the other hand, for $r>0$ and $m \leq 0$, we easily know that the functions $x^{r}$ and $\left(x^{r}+x_{j}^{r}\right)^{1 / r}$ are increasing about $x$. By applying Lemma 21, we have

$$
\begin{gathered}
\left(x_{1}-x_{2}\right)\left(x_{1}^{r}-x_{2}^{r}\right) \geq 0 ; \\
\frac{x_{1}-x_{2}}{u-v}=\frac{\left(x_{1}-x_{2}\right)^{2}}{\left(x_{1}-x_{2}\right)(u-v)} \geq 0 ; \\
(u-v)\left\{\frac{u f^{\prime}(u)}{f(u)} \cdot \frac{\left(u^{r}-x_{j}^{r}\right)^{(r-m) / r}}{u^{r}}\right. \\
\left.-\frac{v f^{\prime}(v)}{f(v)} \cdot \frac{\left(u^{r}-x_{j}^{r}\right)^{(r-m) / r}}{v^{r}}\right\} \geq 0 .
\end{gathered}
$$

Because the function $f$ is increasing and $f \geq 0$, and applying (27)-(28), we obtain $I I \geq 0$. 
Lemma 23. Let $f: \Omega \subset \mathbb{R}_{++}^{n} \rightarrow \mathbb{R}_{++}$be continuous on $\Omega$ and differentiable in the interior of $\Omega$. If $f$ is increasing and multiplicatively convex, then for $m \leq 0$ and $r>0$, one has

$$
\begin{aligned}
& I I I=F_{n, k}(\mathbf{x}, r)\left(x_{1}-x_{2}\right)\left(x_{1}^{r-m}-x_{2}^{r-m}\right) \\
& \times \sum_{2 \leq i_{1}<i_{2}<\cdots<i_{k-2} \leq n} \frac{f^{\prime}\left[\left(x_{1}^{r}+\sum_{j=1}^{k-1} x_{i_{j}}^{r}\right)^{1 / r}\right]}{f\left[\left(x_{1}^{r}+\sum_{j=1}^{k-1} x_{i_{j}}^{r}\right)^{1 / r}\right]} \geq 0, \\
& \begin{aligned}
I V=F_{n, k}(\mathbf{x}, r)\left(x_{1}-x_{2}\right) \\
\sum_{3 \leq i_{1}<i_{2}<\ldots<i_{k-1} \leq n}\left\{\frac{f^{\prime}\left[\left(x_{1}^{r}+\sum_{j=1}^{k-1} x_{i_{j}}^{r}\right)^{1 / r}\right]}{f\left[\left(x_{1}^{r}+\sum_{j=1}^{k-1} x_{i_{j}}^{r}\right)^{1 / r}\right]}\right. \\
\cdot\left(x_{1}^{r}+\sum_{j=1}^{k-1} x_{i_{j}}^{r}\right)^{1 / r-1} \\
\cdot x_{1}^{r-m}-\frac{f^{\prime}\left[\left(x_{2}^{r}+\sum_{j=1}^{k-1} x_{i_{j}}^{r}\right)^{1 / r}\right]}{f\left[\left(x_{2}^{r}+\sum_{j=1}^{k-1} x_{i_{j}}^{r}\right)^{1 / r}\right]} \\
\left.\cdot\left(x_{2}^{r}+\sum_{j=1}^{k-1} x_{i_{j}}^{r}\right)^{1 / r-1} \cdot x_{2}^{r-m}\right\} \geq 0 .
\end{aligned}
\end{aligned}
$$

Proof. Set $u=\left(x_{1}^{r}+\sum_{j=1}^{k-1} x_{i_{j}}^{r}\right)^{1 / r}, v=\left(x_{1}^{r}+\sum_{j=1}^{k-1} x_{i_{j}}^{r}\right)^{1 / r}$; it is easy that $u, v \in \Omega$. Then

$$
\begin{aligned}
& I V= F_{n, k}(\mathbf{x}, r) \frac{x_{1}-x_{2}}{u-v}(u-v) \\
& \times \sum_{3 \leq i_{1}<i_{2}<\cdots<i_{k-1} \leq n}\left\{\frac{u f^{\prime}(u)}{f(u)} \cdot \frac{\left(u^{r}-w\right)^{(r-m) / r}}{u^{r}}\right. \\
& \\
&\left.\quad-\frac{v f^{\prime}(v)}{f(v)} \cdot \frac{\left(v^{r}-w\right)^{(r-m) / r}}{v^{r}}\right\},
\end{aligned}
$$

where $w=\sum_{j=1}^{k-1} x_{i_{j}}^{r}$.

By (27)-(28) and the monotonicity property of the nonnegative function $f$, we get that $I I I \geq 0, I V \geq 0$.

Proof of Theorem 16. By Lemma 10 and Remark 12, we only need to prove that

$$
\left(x_{1}-x_{2}\right)\left(x_{1}^{1-m} \frac{\partial F_{n, k}(\mathbf{x}, r)}{x_{1}}-x_{2}^{1-m} \frac{\partial F_{n, k}(\mathbf{x}, r)}{x_{2}}\right) \geq 0 .
$$

To prove the above inequality, we consider the following three cases for $k$.
Case 1. For $k=1$. It is clear that $F_{n, 1}(\mathbf{x}, r)=\prod_{i=1}^{n} f\left(x_{i}\right)$. From (31), it follows that

$$
\begin{aligned}
\Delta_{1} & =\left(x_{1}-x_{2}\right)\left(x_{1}^{1-m} \frac{\partial F_{n, 1}(\mathbf{x}, r)}{x_{1}}-x_{2}^{1-m} \frac{\partial F_{n, 1}(\mathbf{x}, r)}{x_{2}}\right) \\
& =\left(x_{1}-x_{2}\right)\left(x_{1}^{1-m} \frac{f^{\prime}\left(x_{1}\right)}{f\left(x_{1}\right)}-x_{2}^{1-m} \frac{f^{\prime}\left(x_{2}\right)}{f\left(x_{2}\right)}\right) \prod_{i=1}^{n} f\left(x_{i}\right) .
\end{aligned}
$$

By Lemma 20, and $f(x) \geq 0$, it follows that $\Delta_{1} \geq 0$.

Case 2. For $k=2, r>0$, we have $F_{n, 2}(\mathbf{x}, r)=$ $\prod_{1 \leq i<j \leq n} f\left[\left(x_{i}^{r}+x_{j}^{r}\right)^{1 / r}\right]$. We can easily derive that

$$
\begin{aligned}
\ln F_{n, 2}(\mathbf{x}, r)= & \sum_{1 \leq i<j \leq n} \ln f\left[\left(x_{i}^{r}+x_{j}^{r}\right)^{1 / r}\right] \\
= & \sum_{j=2}^{n} \ln f\left[\left(x_{1}^{r}+x_{j}^{r}\right)^{1 / r}\right] \\
& +\sum_{2 \leq i<j \leq n} \ln f\left[\left(x_{i}^{r}+x_{j}^{r}\right)^{1 / r}\right] .
\end{aligned}
$$

By differentiating the above equation with respect to $x_{1}$, we obtain

$$
\begin{aligned}
& \frac{\partial F_{n, 2}(\mathbf{x}, r)}{x_{1}}=F_{n, 2}(\mathbf{x}, r) \cdot \frac{\partial \ln F_{n, 2}(\mathbf{x}, r)}{x_{1}} \\
&=F_{n, 2}(\mathbf{x}, r) \cdot\left\{\sum_{j=2}^{n} \frac{f^{\prime}\left[\left(x_{1}^{r}+x_{j}^{r}\right)^{1 / r}\right]}{f\left[\left(x_{1}^{r}+x_{j}^{r}\right)^{1 / r}\right]}\right. \\
&\left.\cdot\left(x_{1}^{r}+x_{j}^{r}\right)^{1 / r-1} \cdot x_{1}^{r-1}\right\} \\
&=F_{n, 2}(\mathbf{x}, r)\left\{\frac{f^{\prime}\left[\left(x_{1}^{r}+x_{2}^{r}\right)^{1 / r}\right]}{f\left[\left(x_{1}^{r}+x_{2}^{r}\right)^{1 / r}\right]}\right. \\
& \cdot\left(x_{1}^{r}+x_{2}^{r}\right)^{1 / r-1} \cdot x_{1}^{r-1} \\
&+\sum_{j=3}^{n} \frac{f^{\prime}\left[\left(x_{1}^{r}+x_{j}^{r}\right)^{1 / r}\right]}{f\left[\left(x_{1}^{r}+x_{j}^{r}\right)^{1 / r}\right]} \\
&\left.\cdot\left(x_{1}^{r}+x_{j}^{r}\right)^{1 / r-1} \cdot x_{1}^{r-1}\right\} .
\end{aligned}
$$


Similarly, we have

$$
\begin{aligned}
\frac{\partial F_{n, 2}(\mathbf{x}, r)}{x_{2}}= & F_{n, 2}(\mathbf{x}, r) \\
& \times\left\{\frac{f^{\prime}\left[\left(x_{1}^{r}+x_{2}^{r}\right)^{1 / r}\right]}{f\left[\left(x_{1}^{r}+x_{2}^{r}\right)^{1 / r}\right]} \cdot\left(x_{1}^{r}+x_{2}^{r}\right)^{1 / r-1} \cdot x_{2}^{r-1}\right. \\
& +\sum_{j=3}^{n} \frac{f^{\prime}\left[\left(x_{2}^{r}+x_{j}^{r}\right)^{1 / r}\right]}{f\left[\left(x_{2}^{r}+x_{j}^{r}\right)^{1 / r}\right]} \\
& \left.\cdot\left(x_{2}^{r}+x_{j}^{r}\right)^{1 / r-1} \cdot x_{2}^{r-1}\right\} .
\end{aligned}
$$

So, from (34) and (35), and by applying Lemma 22, we have

$$
\left.\begin{array}{rl}
\Delta_{2}= & \left(x_{1}-x_{2}\right)\left(x_{1}^{1-m} \frac{\partial F_{n, 2}(\mathbf{x}, r)}{x_{1}}-x_{2}^{1-m} \frac{\partial F_{n, 2}(\mathbf{x}, r)}{x_{2}}\right) \\
= & F_{n, 2}(\mathbf{x}, r)\left(x_{1}-x_{2}\right)\left(x_{1}^{r-m}-x_{2}^{r-m}\right) \frac{f^{\prime}\left[\left(x_{1}^{r}+x_{2}^{r}\right)^{1 / r}\right]}{f\left[\left(x_{1}^{r}+x_{2}^{r}\right)^{1 / r}\right]} \\
& \times\left(x_{1}^{r}+x_{2}^{r}\right)^{1 / r-1}+F_{n, 2}(\mathbf{x}, r)\left(x_{1}-x_{2}\right) \\
& \cdot \sum_{j=3}^{n}\left\{\frac{f^{\prime}\left[\left(x_{1}^{r}+x_{j}^{r}\right)^{1 / r}\right]}{f\left[\left(x_{1}^{r}+x_{j}^{r}\right)^{1 / r}\right]} \cdot\left(x_{1}^{r}+x_{j}^{r}\right)^{1 / r-1} \cdot x_{1}^{r-m}\right. \\
= & \quad-\frac{f^{\prime}\left[\left(x_{2}^{r}+x_{j}^{r}\right)^{1 / r}\right]}{f\left[\left(x_{2}^{r}+x_{j}^{r}\right)^{1 / r}\right]} \cdot\left(x_{2}^{r}+x_{j}^{r}\right)^{1 / r-1} \cdot x_{2}^{r-m}
\end{array}\right\}
$$

So we get that $\Delta_{2} \geq 0$.

Case 3. For $3 \leq k \leq n, r>0$, similarly to the discussion of Case 2, we have

$$
\begin{aligned}
\ln F_{n, 2}(\mathbf{x}, r)= & \sum_{1 \leq i_{1}<i_{2}<\cdots<i_{k} \leq n} \ln f\left[\left(\sum_{j=1}^{k} x_{i_{j}}^{r}\right)^{1 / r}\right] \\
= & \sum_{2 \leq i_{1}<i_{2}<\cdots<i_{k-1} \leq n} \ln f\left[\left(x_{1}^{r}+\sum_{j=1}^{k-1} x_{i_{j}}^{r}\right)^{1 / r}\right] \\
& +\sum_{2 \leq i_{1}<i_{2}<\cdots<i_{k} \leq n} \ln f\left[\left(\sum_{j=1}^{k} x_{i_{j}}^{r}\right)^{1 / r}\right] .
\end{aligned}
$$

By differentiating to the above with respect to $x_{1}$, we have

$$
\begin{aligned}
& \frac{\partial F_{n, k}(\mathbf{x}, r)}{x_{1}} \\
& =F_{n, k}(\mathbf{x}, r) \cdot \frac{\partial \ln F_{n, k}(\mathbf{x}, r)}{x_{1}} \\
& =F_{n, k}(\mathbf{x}, r) \cdot \sum_{2 \leq i_{1}<i_{2}<\cdots<i_{k-1} \leq n} \frac{f^{\prime}\left[\left(x_{1}^{r}+\sum_{j=1}^{k-1} x_{i_{j}}^{r}\right)^{1 / r}\right]}{f\left[\left(x_{1}^{r}+\sum_{j=1}^{k-1} x_{i_{j}}^{r}\right)^{1 / r}\right]} \\
& \cdot\left(x_{1}^{r}+\sum_{j=1}^{k-1} x_{i_{j}}^{r}\right)^{1 / r-1} \cdot x_{1}^{r-1} \\
& =F_{n, k}(\mathbf{x}, r)\left\{\sum_{3 \leq i_{1}<i_{2}<\cdots<i_{k-2} \leq n} \frac{f^{\prime}\left[\left(x_{1}^{r}+x_{2}^{r}+\sum_{j=1}^{k-2} x_{i_{j}}^{r}\right)^{1 / r}\right]}{f\left[\left(x_{1}^{r}+x_{2}^{r}+\sum_{j=1}^{k-2} x_{i_{j}}^{r}\right)^{1 / r}\right]}\right. \\
& \left(x_{1}^{r}+x_{2}^{r}+\sum_{j=1}^{k-2} x_{i_{j}}^{r}\right)^{1 / r-1} \cdot x_{1}^{r-1} \\
& +\sum_{3 \leq i_{1}<i_{2}<\cdots<i_{k-1} \leq n} \frac{f^{\prime}\left[\left(x_{1}^{r}+\sum_{j=1}^{k-1} x_{i_{j}}^{r}\right)^{1 / r}\right]}{f\left[\left(x_{1}^{r}+\sum_{j=1}^{k-1} x_{i_{j}}^{r}\right)^{1 / r}\right]} \\
& \left.\cdot\left(x_{1}^{r}+\sum_{j=1}^{k-1} x_{i_{j}}^{r}\right)^{1 / r-1} \cdot x_{1}^{r-1}\right\} .
\end{aligned}
$$

Similarly, we can have

$$
\begin{aligned}
& \frac{\partial F_{n, k}(\mathbf{x}, r)}{x_{2}} \\
& =F_{n, k}(\mathbf{x}, r) \cdot \frac{\partial \ln F_{n, k}(\mathbf{x}, r)}{x_{2}} \\
& =F_{n, k}(\mathbf{x}, r)\left\{\sum_{3 \leq i_{1}<i_{2}<\cdots<i_{k-2} \leq n} \frac{f^{\prime}\left[\left(x_{1}^{r}+x_{2}^{r}+\sum_{j=1}^{k-2} x_{i_{j}}^{r}\right)^{1 / r}\right]}{f\left[\left(x_{1}^{r}+x_{2}^{r}+\sum_{j=1}^{k-2} x_{i_{j}}^{r}\right)^{1 / r}\right]}\right. \\
& \cdot\left(x_{1}^{r}+x_{2}^{r}+\sum_{j=1}^{k-2} x_{i_{j}}^{r}\right)^{1 / r-1} \cdot x_{1}^{r-1} \\
& +\sum_{3 \leq i_{1}<i_{2}<\cdots<i_{k-1} \leq n} \frac{f^{\prime}\left[\left(x_{2}^{r}+\sum_{j=1}^{k-1} x_{i_{j}}^{r}\right)^{1 / r}\right]}{f\left[\left(x_{2}^{r}+\sum_{j=1}^{k-1} x_{i_{j}}^{r}\right)^{1 / r}\right]} \\
& \left.\left(x_{2}^{r}+\sum_{j=1}^{k-1} x_{i_{j}}^{r}\right)^{1 / r-1} \cdot x_{2}^{r-1}\right\}
\end{aligned}
$$


From (38) and (39), we have

$$
\begin{aligned}
& \Delta_{3}=\left(x_{1}-x_{2}\right)\left(x_{1}^{1-m} \frac{\partial F_{n, k}(\mathbf{x}, r)}{x_{1}^{1-m}}-x_{2}^{1-m} \frac{\partial F_{n, k}(\mathbf{x}, r)}{x_{2}}\right) \\
& =F_{n, k}(\mathbf{x}, r)\left(x_{1}-x_{2}\right)\left(x_{1}^{r-m}-x_{2}^{r-m}\right) \\
& \times \sum_{2 \leq i_{1}<i_{2}<\cdots<i_{k-2} \leq n} \frac{f^{\prime}\left[\left(x_{1}^{r}+\sum_{j=1}^{k-1} x_{i_{j}}^{r}\right)^{1 / r}\right]}{f\left[\left(x_{1}^{r}+\sum_{j=1}^{k-1} x_{i_{j}}^{r}\right)^{1 / r}\right]} \\
& +F_{n, k}(\mathbf{x}, r)\left(x_{1}-x_{2}\right) \\
& \times \sum_{3 \leq i_{1}<i_{2}<\cdots<i_{k-1} \leq n}\left\{\frac{f^{\prime}\left[\left(x_{1}^{r}+\sum_{j=1}^{k-1} x_{i_{j}}^{r}\right)^{1 / r}\right]}{f\left[\left(x_{1}^{r}+\sum_{j=1}^{k-1} x_{i_{j}}^{r}\right)^{1 / r}\right]}\right. \\
& \cdot\left(x_{1}^{r}+\sum_{j=1}^{k-1} x_{i_{j}}^{r}\right)^{1 / r-1} \cdot x_{1}^{r-m} \\
& -\frac{f^{\prime}\left[\left(x_{2}^{r}+\sum_{j=1}^{k-1} x_{i_{j}}^{r}\right)^{1 / r}\right]}{f\left[\left(x_{2}^{r}+\sum_{j=1}^{k-1} x_{i_{j}}^{r}\right)^{1 / r}\right]} \\
& \left.\left(x_{2}^{r}+\sum_{j=1}^{k-1} x_{i_{j}}^{r}\right)^{1 / r-1} \cdot x_{2}^{r-m}\right\} \\
& =I I I+I V \geq 0 .
\end{aligned}
$$

So we get that $\Delta_{2} \geq 0$. So the proof of Theorem 16 is complete.

Proof of Theorem 19. Let $x=\left(x_{1}, x_{2}, \ldots, x_{n}\right) \in \mathbb{R}_{++}^{n}$, and $\sum_{i=1}^{n} x_{i}=s$. From $[13,14]$, we have

$$
\begin{aligned}
\frac{s-\mu x}{n-\mu} & =\left(\frac{s-\mu x_{1}}{n-\mu}, \frac{s-\mu x_{2}}{n-\mu}, \ldots, \frac{s-\mu x_{n}}{n-\mu}\right) \\
& \prec\left(x_{1}, x_{2}, \ldots, x_{n}\right)=x, \\
\frac{s+\mu x}{n+\mu} & =\left(\frac{s+\mu x_{1}}{n+\mu}, \frac{s+\mu x_{2}}{n+\mu}, \ldots, \frac{s+\mu x_{n}}{n+\mu}\right) \\
& \prec\left(x_{1}, x_{2}, \ldots, x_{n}\right)=x .
\end{aligned}
$$
(21).

From (41), and contacting Corollaries 17 and 18, we get

\section{Applications}

Let $x_{i}>0, i=1,2, \ldots, n$ be $n$ positive real numbers and set $\mathbf{x}=\left\{x_{1}, x_{2}, \ldots, x_{n}\right\}$. The $p$ th power mean of of order $p \in \mathbb{R}$ of $x_{i}$ is defined by

$$
M_{p}(\mathbf{x})= \begin{cases}\left(\frac{x_{1}^{p}+x_{2}^{p}+\cdots+x_{n}^{p}}{n}\right)^{1 / p}, & p \neq 0 \\ \sqrt{x_{1} x_{2} \cdots x_{n}}, & p=0 .\end{cases}
$$

In particular, for $p=1, p=0$, and $p=1$ we, respectively, get the arithmetic, the geometric, and the harmonic means of $x_{i}$, and set $A_{n}(\mathbf{x})=M_{1}(\mathbf{x})=(1 / n) \sum_{i=1}^{n} x_{i}, G_{n}(\mathbf{x})=M_{0}(\mathbf{x})=$ $\left(\prod_{i=1}^{n} x_{i}\right)^{1 / n}, H_{n}(\mathbf{x})=M_{-1}(\mathbf{x})=n / \sum_{i=1}^{n}\left(1 / x_{i}\right)$.

In this section, some applications of the results in Section 3 are given. Some analytic inequalities are established. In particular, several inequalities involving the $p$ th power mean and the arithmetic, the geometric, or the harmonic means are presented.

4.1. Analytic Inequalities. To establish some analytic inequalities, we first give a lemma.

Lemma 24 (see [20]). Let $x_{i}>0, i=1,2, \ldots, n$. Then the following relations is known:

$$
\begin{aligned}
\left(A_{n}(\mathbf{x}), A_{n}(\mathbf{x}), \ldots, A_{n}(\mathbf{x})\right) & \prec\left(x_{1}, x_{2}, \ldots, x_{n}\right) ; \\
\ln \left(G_{n}(\mathbf{x}), G_{n}(\mathbf{x}), \ldots, G_{n}(\mathbf{x})\right) & \prec \ln \left(x_{1}, x_{2}, \ldots, x_{n}\right) ; \\
\left(\frac{1}{H_{n}(\mathbf{x})}, \frac{1}{H_{n}(\mathbf{x})}, \ldots, \frac{1}{H_{n}(\mathbf{x})}\right) & \prec\left(\frac{1}{x_{1}}, \frac{1}{x_{2}}, \ldots, \frac{1}{x_{n}}\right) .
\end{aligned}
$$

By applying Lemma 24 and Theorem 16, Corollaries 17 and 18, we can get the following several theorems and corollaries.

Theorem 25. For $r>0$ and $k=1,2, \ldots, n$, the symmetric functions

$$
U_{k}(\mathbf{x}, r)=\prod_{1 \leq i_{1}<i_{2}<\cdots<i_{k} \leq n} \tan \left(\sum_{j=1}^{k} x_{i_{j}}^{r}\right)^{1 / r}
$$

are Schur $m$-power convex in $\mathbb{R}_{++}^{n}$ when $m \leq 0$. In particular, $U_{k}(\mathbf{x}, r)$ are Schur geometrically convex and Schur harmonically convex in $\mathbb{R}_{++}^{n}$.

Proof. For $x \in \mathbb{R}_{++}$, let $f(x)=\tan x$. Then $f^{\prime}(x)=1 / \cos ^{2} x=$ $\sec ^{2} x \geq 0$ and

$$
\begin{gathered}
x\left[f(x) f^{\prime \prime}(x)-\left(f^{\prime}(x)\right)^{2}\right]+f(x) f^{\prime}(x) \\
=\sec ^{2} x\left(x \sec ^{2} x-2 x+\tan x\right) .
\end{gathered}
$$

Let $g(x)=x \sec ^{2} x-2 x+\tan x$; then $g^{\prime}(x)=2(1+$ $x \tan x) \sec ^{2} x-2 \geq 0$, and $g(x)$ is increasing. From the monotonicity of $g(x)$, we get that

$$
\begin{gathered}
x\left[f(x) f^{\prime \prime}(x)-\left(f^{\prime}(x)\right)^{2}\right]+f(x) f^{\prime}(x) \\
=\sec ^{2} x g(x) \geq g(0)=0 .
\end{gathered}
$$

Therefore, $f(x)$ is increasing and multiplicatively convex in $\mathbb{R}_{++}^{n}$. By applying Theorem 16 , we obtain the result.

By using Theorem 25 and Lemma 24, we get the following inequalities. 
Corollary 26. For $\mathbf{x} \in \mathbb{R}_{++}^{n}$, then

$$
\begin{gathered}
\prod_{i=1}^{n}\left(\tan \frac{1}{x_{i}}\right)^{1 / n} \geq \tan \left(\frac{1}{A(\mathbf{x})}\right) ; \\
\prod_{i=1}^{n}\left(\tan x_{i}\right)^{1 / n} \geq \tan G(\mathbf{x}) ; \\
\prod_{i=1}^{n}\left(\tan x_{i}\right)^{1 / n} \geq \tan H(\mathbf{x}) .
\end{gathered}
$$

Theorem 27. For $r>0$ and $k=1,2, \ldots, n$, the symmetric functions

$$
V_{k}(\mathbf{x}, r)=\prod_{1 \leq i_{1}<i_{2}<\cdots<i_{k} \leq n} \arcsin \left(\sum_{j=1}^{k} x_{i_{j}}^{r}\right)^{1 / r}
$$

are Schur $m$-power convex in $(0,1)^{n}$ when $m \leq 0$. In particular, $V_{k}(\mathbf{x}, r)$ are Schur geometrically convex and Schur harmonically convex in $(0,1)^{n}$.

Proof. For $x \in(0,1)$, let $f(x)=\arcsin x$. Then $f^{\prime}(x)=$ $1 / \sqrt{1-x^{2}} \geq 0$ and

$$
\begin{gathered}
x\left[f(x) f^{\prime \prime}(x)-\left(f^{\prime}(x)\right)^{2}\right]+f(x) f^{\prime}(x) \\
\quad=\frac{\arcsin x-x \sqrt{1-x^{2}}}{\left(1-x^{2}\right) \sqrt{1-x^{2}}} .
\end{gathered}
$$

Let $h(x)=\arctan x-x^{2} \arctan x-x$; then $h^{\prime}(x)=$ $2 x^{2} / \sqrt{1-x^{2}} \geq 0$, and $h(x)$ is increasing. From the monotonicity of $h(x)$, we get that

$$
\begin{gathered}
x\left[f(x) f^{\prime \prime}(x)-\left(f^{\prime}(x)\right)^{2}\right]+f(x) f^{\prime}(x) \\
=\frac{h(x)}{\left(1-x^{2}\right) \sqrt{1-x^{2}}} \geq h(0)=0 .
\end{gathered}
$$

Therefore, $f(x)$ is increasing and multiplicatively convex in $\mathbb{R}_{++}^{n}$. By applying Theorem 16 , we obtain the result.

By using Theorem 27 and Lemma 24, we get the following inequalities.

Corollary 28. For $\mathbf{x} \in(0,1)^{n}$, then

$$
\begin{gathered}
\prod_{i=1}^{n}\left(\arcsin \frac{1}{x_{i}}\right)^{1 / n} \geq \arcsin \left(\frac{1}{A(\mathbf{x})}\right) ; \\
\prod_{i=1}^{n}\left(\arcsin x_{i}\right)^{1 / n} \geq \arcsin G(\mathbf{x}) ; \\
\prod_{i=1}^{n}\left(\arcsin x_{i}\right)^{1 / n} \geq \arcsin H(\mathbf{x}) .
\end{gathered}
$$

Theorem 29. For $r>0$ and $k=1,2, \ldots, n$, the symmetric functions

$$
M_{k}(\mathbf{x}, r)=\prod_{1 \leq i_{1}<i_{2}<\cdots<i_{k} \leq n}\left(\sum_{j=1}^{k} x_{i_{j}}^{r}\right)^{1 / r}
$$

are Schur $m$-power convex in $\mathbb{R}_{++}^{n}$ when $m \leq 0$. In particular, $M_{k}(\mathbf{x}, r)$ are Schur geometrically convex in $\mathbb{R}_{++}^{n}$ and Schur harmonically convex in $\mathbb{R}_{++}^{n}$.

Proof. Let $f(x)=x, x \in \mathbb{R}_{++}$. We can easily see that $f(x)$ is increasing and multiplicatively convex in $\mathbb{R}_{++}$. By applying Theorem 16, we obtain the result.

By using Theorem 29 and Lemma 24, we obtain the following results.

Corollary 30. Let $\mathbf{x} \in \mathbb{R}_{++}$, for $r>0$ and $k=1,2, \ldots, n$; one has

$$
\begin{gathered}
\left(k^{1 / r} \frac{1}{A_{n}(\mathbf{x})}\right)^{C_{n}^{k}} \leq \prod_{1 \leq i_{1}<i_{2}<\cdots<i_{k} \leq n}\left(\sum_{j=1}^{k} \frac{1}{x_{i_{j}}^{r}}\right)^{1 / r}, \\
\left(k^{1 / r} G_{n}(\mathbf{x})\right)^{C_{n}^{k}} \leq \prod_{1 \leq i_{1}<i_{2}<\cdots<i_{k} \leq n}\left(\sum_{j=1}^{k} x_{i_{j}}^{r}\right)^{1 / r}, \\
\left(k^{1 / r} H_{n}(\mathbf{x})\right)^{C_{n}^{k}} \leq \prod_{1 \leq i_{1}<i_{2}<\cdots<i_{k} \leq n}\left(\sum_{j=1}^{k} x_{i_{j}}^{r}\right)^{1 / r} .
\end{gathered}
$$

In particular, take $k=1$ in (53); we get the known A-G$\mathrm{H}$ inequality. And take $k=n$ in (53); we get the following inequalities:

$$
\left(\sum_{i=1}^{n} \frac{1}{x_{i}^{r}}\right)^{1 / r} \cdot \sum_{i=1}^{n} x_{i} \geq n^{1 / r+1}
$$

Theorem 31. For $r>0$ and $k=1,2, \ldots, n$, the symmetric functions

$$
N_{k}(\mathbf{x}, r)=\prod_{1 \leq i_{1}<i_{2}<\cdots<i_{k} \leq n} \frac{\left(\sum_{j=1}^{k} x_{i_{j}}^{r}\right)^{1 / r}}{1-\left(\sum_{j=1}^{k} x_{i_{j}}^{k}\right)^{1 / r}}
$$

are Schur $m$-power convex on $(0,1)^{n}$ when $m \leq 0$. In particular, $N_{k}(\mathbf{x}, r)$ are Schur geometrically convex on $(0,1)^{n}$ and Schur harmonically convex on $(0,1)^{n}$.

Proof. Let $f(x)=x /(1-x), x \in(0,1)$. Then $f^{\prime}(x)=$ $\left(1 /(1-x)^{2}\right) \geq 0$, and

$$
x\left[f(x) f^{\prime \prime}(x)-f^{\prime 2}(x)\right]+f(x) f^{\prime}(x)=\frac{x^{2}}{(1-x)^{4}} \geq 0 .
$$

So $f(x)$ is increasing and multiplicatively convex in $(0,1)$. By applying Theorem 16, we obtain the result.

By using Theorem 31 and Lemma 24, we obtain the following results. 
Corollary 32. Let $\mathbf{x} \in(0,1)^{n}$, for $r>0$ and $k=1,2, \ldots, n$, one has

$$
\begin{gathered}
\left(\frac{k^{1 / r} G_{n}(\mathbf{x})}{1-k^{1 / r} G_{n}(\mathbf{x})}\right)^{C_{n}^{k}} \leq \prod_{1 \leq i_{1}<i_{2}<\cdots<i_{k} \leq n} \frac{\left(\sum_{j=1}^{k} x_{i_{j}}^{r}\right)^{1 / r}}{1-\left(\sum_{j=1}^{k} x_{i_{j}}^{r}\right)^{1 / r}}, \\
\left(\frac{k^{1 / r} H_{n}(\mathbf{x})}{1-k^{1 / r} H_{n}(\mathbf{x})}\right)^{C_{n}^{k}} \leq \prod_{1 \leq i_{1}<i_{2}<\cdots<i_{k} \leq n} \frac{\left(\sum_{j=1}^{k} x_{i_{j}}^{r}\right)^{1 / r}}{1-\left(\sum_{j=1}^{k} x_{i_{j}}^{r}\right)^{1 / r}} .
\end{gathered}
$$

In particular, take $k=1$ or $k=n$ in (59), we get the following inequalities:

$$
\begin{gathered}
\left(\frac{G_{n}(\mathbf{x})}{1-G_{n}(\mathbf{x})}\right)^{n} \leq \prod_{i=1}^{n} \frac{x_{i}}{1-x_{i}}, \\
\left(\frac{H_{n}(\mathbf{x})}{1-H_{n}(\mathbf{x})}\right)^{n} \leq \prod_{i=1}^{n} \frac{x_{i}}{1-x_{i}}, \\
\frac{G_{n}(\mathbf{x})}{1-n^{1 / r} G_{n}(\mathbf{x})} \leq \frac{M_{r}(\mathbf{x})}{1-n^{1 / r} M_{r}(\mathbf{x})}, \\
\frac{H_{n}(\mathbf{x})}{1-n^{1 / r} H_{n}(\mathbf{x})} \leq \frac{M_{r}(\mathbf{x})}{1-n^{1 / r} M_{r}(\mathbf{x})} .
\end{gathered}
$$

Theorem 33. For $r>0$ and $k=1,2, \ldots, n$, the symmetric functions

$$
I_{k}(\mathbf{x}, r)=\prod_{1 \leq i_{1}<i_{2}<\cdots<i_{k} \leq n} \frac{1+\left(\sum_{j=1}^{k} x_{i_{j}}^{r}\right)^{1 / r}}{1-\left(\sum_{j=1}^{k} x_{i_{j}}^{r}\right)^{1 / r}}
$$

are Schur $m$-power convex on $(0,1)^{n}$ when $m \leq 0$. In particular, $I_{k}(\mathbf{x}, r)$ are Schur geometrically convex and Schur harmonically convex on $(0,1)^{n}$.

Proof. Let $f(x)=x /(1-x), x \in(0,1)$. Then $f^{\prime}(x)=$ $\left(2 /(1-x)^{2}\right) \geq 0$, and

$$
x\left[f(x) f^{\prime \prime}(x)-f^{\prime 2}(x)\right]+f(x) f^{\prime}(x)=\frac{2-x^{2}}{(1-x)^{4}} \geq 0 .
$$

So $f(x)$ is increasing and multiplicatively convex in $(0,1)$. By applying Theorem 16, we obtain the result.

By using Theorem 33 and Lemma 24, we get the following inequalities.

Corollary 34. Let $\mathbf{x} \in(0,1)^{n}$, for $r>0$ and $k=1,2, \ldots, n$; one has

$$
\begin{gathered}
\left(\frac{1+k^{1 / r} G_{n}(\mathbf{x})}{1-k^{1 / r} G_{n}(\mathbf{x})}\right)^{C_{n}^{k}} \leq \prod_{1 \leq i_{1}<i_{2}<\cdots<i_{k} \leq n} \frac{1+\left(\sum_{j=1}^{k} x_{i_{j}}^{r}\right)^{1 / r}}{1-\left(\sum_{j=1}^{k} x_{i_{j}}^{r}\right)^{1 / r}}, \\
\left(\frac{1+k^{1 / r} H_{n}(\mathbf{x})}{1-k^{1 / r} H_{n}(\mathbf{x})}\right)^{C_{n}^{k}} \leq \prod_{1 \leq i_{1}<i_{2}<\cdots<i_{k} \leq n} \frac{1+\left(\sum_{j=1}^{k} x_{i_{j}}^{r}\right)^{1 / r}}{1-\left(\sum_{j=1}^{k} x_{i_{j}}^{r}\right)^{1 / r}} .
\end{gathered}
$$

In particular, take $k=1$ or $k=n$ in (63); we get the following inequalities:

$$
\begin{gathered}
\left(\frac{1+G_{n}(\mathbf{x})}{1-G_{n}(\mathbf{x})}\right)^{n} \leq \prod_{i=1}^{n} \frac{1+x_{i}}{1-x_{i}}, \\
\left(\frac{1+H_{n}(\mathbf{x})}{1-H_{n}(\mathbf{x})}\right)^{n} \leq \prod_{i=1}^{n} \frac{1+x_{i}}{1-x_{i}}, \\
\frac{1+n^{1 / r} G_{n}(\mathbf{x})}{1-n^{1 / r} G_{n}(\mathbf{x})} \leq \frac{1+n^{1 / r} M_{r}(\mathbf{x})}{1-n^{1 / r} M_{r}(\mathbf{x})}, \\
\frac{1+n^{1 / r} H_{n}(\mathbf{x})}{1-n^{1 / r} H_{n}(\mathbf{x})} \leq \frac{1+n^{1 / r} M_{r}(\mathbf{x})}{1-n^{1 / r} M_{r}(\mathbf{x})} .
\end{gathered}
$$

Theorem 35. For $r>0$ and $k=1,2, \ldots, n$, the symmetric functions

$$
T_{k}(\mathbf{x}, r)=\prod_{1 \leq i_{1}<i_{2}<\cdots<i_{k} \leq n}\left[\left(\sum_{j=1}^{k} x_{i_{j}}^{r}\right)^{1 / r}\right]^{\left(\sum_{j=1}^{r} x_{i_{j}}^{r}\right)^{1 / r}}
$$

are Schur m-power convex on $\left(e^{-1},+\infty\right)^{n}$ when $m \leq 0$. In particular, $T_{k}(\mathbf{x}, r)$ are Schur geometrically convex and Schur harmonically convex on $\left(e^{-1},+\infty\right)^{n}$.

Proof. Let $f(x)=x^{x}, x \in\left(e^{-1},+\infty\right)$. Then $f^{\prime}(x)=x^{x}(\ln x+$ 1) $\geq 0$, and

$$
\begin{gathered}
x\left[f(x) f^{\prime \prime}(x)-f^{\prime 2}(x)\right]+f(x) f^{\prime}(x) \\
=f^{2}(x)(\ln x+2) \geq 0 .
\end{gathered}
$$

So $f(x)$ is increasing and multiplicatively convex on $\left(e^{-1},+\infty\right)$. By applying Theorem 16 , we obtain the result.

By using Theorem 35 and Lemma 24, we get the following inequalities.

Corollary 36. Let $\mathbf{x} \in\left(e^{-1},+\infty\right)^{n}$, for $r>0$ and $k=$ $1,2, \ldots, n$; one has

$$
\begin{aligned}
& \left(k^{1 / r} \frac{1}{A_{n}(\mathbf{x})}\right)^{k^{1 / r}\left(1 / A_{n}(\mathbf{x})\right) C_{n}^{k}} \\
& \leq \prod_{1 \leq i_{1}<i_{2}<\cdots<i_{k} \leq n}\left(\sum_{j=1}^{k} \frac{1}{x_{i_{j}}^{r}}\right)^{(1 / r)\left(\sum_{j=1}^{k}\left(1 / x_{i_{j}}^{r}\right)\right)^{1 / r}}, \\
& \left(k^{1 / r} G_{n}(\mathbf{x})\right)^{k^{1 / r} G_{n}(\mathbf{x}) C_{n}^{k}} \\
& \leq \prod_{1 \leq i_{1}<i_{2}<\cdots<i_{k} \leq n}\left(\sum_{j=1}^{k} x_{i_{j}}^{r}\right)^{(1 / r)\left(\sum_{j=1}^{k} x_{i_{j}}^{r}\right)^{1 / r},}
\end{aligned}
$$




$$
\begin{aligned}
& \left(k^{1 / r} H_{n}(\mathbf{x})\right)^{k^{1 / r} H_{n}(\mathbf{x}) C_{n}^{k}} \\
& \quad \leq \prod_{1 \leq i_{1}<i_{2}<\cdots<i_{k} \leq n}\left(\sum_{j=1}^{k} x_{i_{j}}^{r}\right)^{(1 / r)\left(\sum_{j=1}^{k} x_{i_{j}}^{r}\right)^{1 / r}} .
\end{aligned}
$$

In particular, take $k=1$ or $k=n$ in (67); we get the following inequalities:

$$
\begin{gathered}
\left(\frac{1}{A_{n}(\mathbf{x})}\right)^{n / A_{n}(\mathbf{x})} \leq \prod_{i=1}^{n}\left(\frac{1}{x_{i}}\right)^{1 / x_{i}}, \\
\left(G_{n}(\mathbf{x})\right)^{n \cdot G_{n}(\mathbf{x})} \leq \prod_{i=1}^{n} x_{i}^{x_{i}}, \\
\left(H_{n}(\mathbf{x})\right)^{n \cdot H_{n}(\mathbf{x})} \leq \prod_{i=1}^{n} x_{i}^{x_{i}}, \\
\left(n^{1 / r} \frac{1}{A_{n}(\mathbf{x})}\right)^{n^{1 / r}\left(1 / A_{n}(\mathbf{x})\right)} \leq\left(n^{1 / r} M_{r}\left(\frac{1}{\mathbf{x}}\right)\right)^{n^{1 / r} M_{r}(1 / \mathbf{x})} \\
\left(n^{1 / r} G_{n}(\mathbf{x})\right)^{n^{1 / r} G_{n}(\mathbf{x})} \leq\left(n^{1 / r} M_{r}(\mathbf{x})\right)^{n^{1 / r} M_{r}(\mathbf{x})}, \\
\left(n^{1 / r} H_{n}(\mathbf{x})\right)^{n^{1 / r} H_{n}(\mathbf{x})} \leq\left(n^{1 / r} M_{r}(\mathbf{x})\right)^{n^{1 / r} M_{r}(\mathbf{x})} .
\end{gathered}
$$

4.2. Geometric Inequalities. In this section, some geometric inequalities of $n$-dimensional simplex are established by use of the results of Theorem 16 .

Lots of geometric inequalities for an $n$-dimensional simplex are established (see [37-42]). In this section, applying the above Lemma and the main results in Section 2, we establish some interesting geometric inequalities on $n$-dimensional simplex in $n$-dimensional Euclidean space $E^{n}$.

In what follows, Let $\Omega=\left\{A_{1}, A_{2}, \ldots, A_{n+1}\right\}$ be an $n$-dimensional simplex in $n$-dimensional Euclidean space $E^{n}(n \geq 2)$ with $V$ the volume. We denote by $h_{i}, r_{i}$, $F_{i}(i=1,2, \ldots, n+1)$, and $r$ the altitudes, the radii of excircles, the areas of lateral surfaces, and the inradius of $\Omega$, respectively. For a given point $P$ in $\Omega$, let $B_{i}$ stand for the intersection point of straight line $A_{i} P$ and hyperplane $a_{i}=\left\{A_{1} \cdots A_{i-1} A_{i+1} \cdots A_{n+1}\right\}$.

We first give some lemmas.

Lemma 37. Let $\Omega=\left\{A_{1}, A_{2}, \ldots, A_{n+1}\right\}$ be an $n$-dimensional simplex in $n$-dimensional Euclidean space $E^{n}(n \geq 2)$. The following relation is

(1) $\left(\frac{1}{n+1}, \frac{1}{n+1}, \ldots, \frac{1}{n+1}\right) \prec\left(\frac{r}{h_{1}}, \frac{r}{h_{2}}, \ldots, \frac{r}{h_{n+1}}\right)$;

(2) $\left(\frac{n-1}{n+1}, \frac{n-1}{n+1}, \ldots, \frac{n-1}{n+1}\right) \prec\left(\frac{r}{r_{1}}, \frac{r}{r_{2}}, \ldots, \frac{r}{r_{n+1}}\right)$;

$$
\text { (3) } \begin{aligned}
& \left(\frac{1}{n+1}, \frac{1}{n+1}, \ldots, \frac{1}{n+1}\right) \\
\text { (4) } & \prec\left(\frac{1}{n+1}, \frac{1}{n+1}, \ldots, \frac{1}{n+1}\right) \\
& \prec\left(\frac{\left|P B_{1}\right|}{\left|A_{1} B_{1}\right|}, \frac{\left|P B_{2}\right|}{\left|A_{2} B_{2}\right|}, \ldots, \frac{\left|P B_{n+1}\right|}{h_{2}+2 r_{2}}, \ldots, \frac{r_{n+1}}{h_{n+1}+2 r_{n+1}}\right) ;
\end{aligned}
$$

where $F=\sum_{i=1}^{n+1} F_{i}$.

Proof. By the formula $[37,38]$

$$
F_{i} h_{i}=n V, \quad \sum_{i=1}^{n+1} F_{i} r=n V, \quad \sum_{i=1}^{n+1}\left(F-2 F_{i}\right) r_{i}=n V
$$

where $F=\sum_{i=1}^{n+1} F_{i}$. From these we get

$$
\begin{gathered}
\sum_{i=1}^{n+1} \frac{r}{h_{i}}=\sum_{i=1}^{n+1} \frac{F_{i} r}{F_{i} h_{i}}=\frac{\sum_{i=1}^{n+1} F_{i} r}{n V}=\frac{n V}{n V}=1, \\
\sum_{i=1}^{n+1} \frac{r}{r_{i}}=\sum_{i=1}^{n+1} \frac{\left(F-2 F_{i}\right) r}{\left(F-2 F_{i}\right) r_{i}} \\
=\frac{\sum_{i=1}^{n+1}\left(F-2 F_{i}\right) r}{n V}=\frac{(n-1) n V}{n V}=n-1, \\
\sum_{i=1}^{n+1} \frac{r_{i}}{h_{i}+2 r_{i}}=\sum_{i=1}^{n+1} \frac{\left(F-2 F_{i}\right) F_{i} r_{i}}{\left(F-2 F_{i}\right) F_{i}\left(h_{i}+2 r_{i}\right)} \\
=\sum_{i=1}^{n+1} \frac{n V F_{i}}{n V\left(F-2 F_{i}\right)+2 n V F_{i}}=\sum_{i=1}^{n+1} \frac{F_{i}}{F}=1 .
\end{gathered}
$$

By Definition 3, and using (74), (75), and (76), respectively, we obtain (69), (70), and (71). In addition, (72) follows from Definition 3 and the fact that $\sum_{i=1}^{n+1}\left|P B_{i}\right| /\left|A_{i} B_{i}\right|=1$. So the proof is complete.

Applying Lemma 37 and Theorem 29, we establish some interesting geometric inequalities on $n$-dimensional simplex in $n$-dimensional Euclidean space $E^{n}$.

Theorem 38. Let $\Omega=\left\{A_{1}, A_{2}, \ldots, A_{n+1}\right\}$ be an $n$ dimensional simplex in $n$-dimensional Euclidean space $E^{n}(n \geq$ 2). For $t>0$ and $k=1,2, \ldots, n$, one has

$$
\begin{aligned}
& \left(r \cdot k^{1 / t}(n+1)\right)^{C_{n+1}^{k}} \leq \prod_{1 \leq i_{1}<i_{2}<\cdots<i_{k} \leq n}\left(\sum_{j=1}^{k} h_{i_{j}}^{t}\right)^{1 / t} ; \\
& \left(r \cdot k^{1 / t} \frac{n+1}{n-1}\right)^{C_{n+1}^{k}} \leq \prod_{1 \leq i_{1}<i_{2}<\cdots<i_{k} \leq n}\left(\sum_{j=1}^{k} r_{i_{j}}^{t}\right)^{1 / t} ;
\end{aligned}
$$




$$
\begin{aligned}
& \left(r \cdot k^{1 / t}(n+1)\right)^{C_{n+1}^{k}} \leq \prod_{1 \leq i_{1}<i_{2}<\cdots<i_{k} \leq n}\left[\sum_{j=1}^{k}\left(\frac{h_{i_{j}}+2 r_{i_{j}}}{r_{i_{j}}}\right)^{t}\right]^{1 / t} ; \\
& \left(r \cdot k^{1 / t}(n+1)\right)^{C_{n+1}^{k}} \leq \prod_{1 \leq i_{1}<i_{2}<\cdots<i_{k} \leq n}\left[\sum_{j=1}^{k}\left(\frac{\left|P A_{i_{j}}\right|}{\left|P B_{i_{j}}\right|}\right)^{t}\right]^{1 / t} .
\end{aligned}
$$

In particular, take $k=1$ in (77), we get the following inequality

$$
\begin{gathered}
r(n+1) \leq \prod_{i=1}^{n+1} h_{i}^{1 /(n+1)} ; \quad r\left(\frac{n+1}{n-1}\right) \leq \prod_{i=1}^{n+1} r_{i}^{1 /(n+1)} ; \\
(n+1) \leq \prod_{i=1}^{n+1}\left(\frac{h_{i}}{r_{i}}+2\right)^{1 /(n+1)} ; \\
(n+1) \leq \prod_{i=1}^{n+1}\left(\frac{\left|P B_{i}\right|}{P A_{i} \mid}\right)^{1 /(n+1)} .
\end{gathered}
$$

\section{Conflict of Interests}

The authors declare that there is no conflict of interests regarding the publication of this paper.

\section{Acknowledgment}

The authors wish to thank the anonymous referees for their careful reading of the paper and their suggestions. This work is supported by the Doctoral Programs Foundation of Education Ministry of China (20113401110009); Universities Natural Science Foundation of Anhui Province (KJ2013A220); and Natural Science Research Project of Hefei Normal University (2012kj11).

\section{References}

[1] P. S. Bullen, D. S. Mitrinovic, and M. Vasic, Handbook of Means and Theirs Inequality, Kluwer Academic, Dordrecht, The Netherlands, 2003.

[2] T. Hara, M. Uchiyama, and S.-E. Takahasi, "A refinement of various mean inequalities," Journal of Inequalities and Applications, vol. 2, no. 4, pp. 387-395, 1998.

[3] K. Z. Guan, "A class of symmetric functions for multiplicatively convex function," Mathematical Inequalities \& Applications, vol. 10, no. 4, pp. 745-753, 2007.

[4] I. Rovenţa, "Schur convexity of a class of symmetric functions," Annals of the University of Craiova. Mathematics and Computer Science Series, vol. 37, no. 1, pp. 12-18, 2010.

[5] J. X. Meng, Y. M. Chu, and X. M. Tang, "The Schur-harmonicconvexity of dual form of the Hamy symmetric function," Matematichki Vesnik, vol. 62, no. 1, pp. 37-46, 2010.

[6] H. N. Shi and J. Zhang, "Schur-convexity, Schur-geometric and Harmonic convexity of dual form of a class symmetric functions," Journal of Inequalities and Applications, vol. 2013, p. 295, 2013.
[7] Zh.-H. Yang, "Schur power convexity of Stolarsky means," Publicationes Mathematicae Debrecen, vol. 80, no. 1-2, pp. 4366, 2012.

[8] Zh.-H. Yang, "Schur power convexity of Gini means," Bulletin of the Korean Mathematical Society, vol. 50, no. 2, pp. 485-498, 2013.

[9] Zh.-H. Yang, "Schur power convexity of the Daróczy means," Mathematical Inequalities \& Applications, vol. 16, no. 3, pp. 751762, 2013.

[10] W. Wang and S. G. Yang, "Schur m-power convexity of generalized Hamy symmetric function," Journal of Mathematical Inequalities. In press.

[11] W. Wang and S. G. Yang, "On the Schur m-power convexity for a class of symmetric functions," Journal of Systems Science and Mathematical Sciences. In press.

[12] B. Y. Wang, Foundations of Majorization Inequalities (in Chinese), Beijing Normal University Press, Beijing, China, 1990.

[13] A. W. Marshall, I. Olkin, and B. C. Arnold, Inequalities: Theory of Majorization and Its Applications, Springer, New York, NY, USA, 2nd edition, 2011.

[14] X. M. Zhang, Geometrically-Convex Functions (in Chinese), Anhui University Press, Hefei, China, 2004.

[15] Y. M. Chu and T. C. Sun, "The Schur harmonic convexity for a class of symmetric functions," Acta Mathematica Scientia B, vol. 30, no. 5, pp. 1501-1506, 2010.

[16] Y. Wu and F. Qi, "Schur-harmonic convexity for differences of some means," Analysis, vol. 32, no. 4, pp. 263-270, 2012.

[17] C. P. Niculescu, "Convexity according to the geometric mean," Mathematical Inequalities \& Applications, vol. 3, no. 2, pp. 155$167,2000$.

[18] B. Y. Long and Y. M. Chu, "The Schur convexity and inequalities for a class of symmetric functions," Acta Mathematica Scientia A, vol. 32, no. 1, pp. 80-89, 2012 (Chinese).

[19] S. H. Wu, "Generalization and sharpness of the power means inequality and their applications," Journal of Mathematical Analysis and Applications, vol. 312, no. 2, pp. 637-652, 2005.

[20] W. F. Xia and Y. M. Chu, "Schur convexity with respect to a class of symmetric functions and their applications," Bulletin of Mathematical Analysis and Applications, vol. 3, no. 3, pp. 84-96, 2011.

[21] H.-T. Ku, M.-C. Ku, and X.-M. Zhang, "Inequalities for symmetric means, symmetric harmonic means, and their applications," Bulletin of the Australian Mathematical Society, vol. 56, no. 3, pp. 409-420, 1997.

[22] K. Z. Guan, "The Hamy symmetric function and its generalization," Mathematical Inequalities \& Applications, vol. 9, no. 4, pp. 797-805, 2006

[23] G. H. Hardy, J. E. Littlewood, and G. Pólya, "Some simple inequalities satisfied by convex functions," Messenger of Mathematics, vol. 58, pp. 145-152, 1929.

[24] Y. M. Chu, W. F. Xia, and T. H. Zhao, "Schur convexity for a class of symmetric functions," Science China. Mathematics, vol. 53, no. 2, pp. 465-474, 2010.

[25] F. Qi, J. Sándor, S. S. Dragomir, and A. Sofo, "Notes on the Schurconvexity of the extended mean values," Taiwanese Journal of Mathematics, vol. 9, no. 3, pp. 411-420, 2005.

[26] G. D. Anderson, M. K. Vamanamurthy, and M. Vuorinen, "Generalized convexity and inequalities," Journal of Mathematical Analysis and Applications, vol. 335, no. 2, pp. 1294-1308, 2007. 
[27] D.-M. Li and H.-N. Shi, "Schur convexity and Schurgeometrically concavity of generalized exponent mean," Journal of Mathematical Inequalities, vol. 3, no. 2, pp. 217-225, 2009.

[28] S. Toader and G. Toader, "Complementaries of Greek means with respect to Gini means," International Journal of Applied Mathematics \& Statistics, vol. 11, no. N07, pp. 187-192, 2007.

[29] W.-F. Xia and Y.-M. Chu, "Schur-convexity for a class of symmetric functions and its applications," Journal of Inequalities and Applications, vol. 2009, Article ID 493759, 15 pages, 2009.

[30] M. Shaked, J. G. Shanthikumar, and Y. L. Tong, "Parametric Schur convexity and arrangement monotonicity properties of partial sums," Journal of Multivariate Analysis, vol. 53, no. 2, pp. 293-310, 1995.

[31] H.-N. Shi, Y.-M. Jiang, and W.-D. Jiang, "Schur-convexity and Schur-geometrically concavity of Gini means," Computers \& Mathematics with Applications, vol. 57, no. 2, pp. 266-274, 2009.

[32] A. Forcina and A. Giovagnoli, "Homogeneity indices and Schur-convex functions," Statistica, vol. 42, no. 4, pp. 529-542, 1982.

[33] Zh.-H. Yang, "Necessary and sufficient condition for Schur convexity of the two-parameter symmetric homogeneous means," Applied Mathematical Sciences, vol. 5, no. 61-64, pp. 3183-3190, 2011.

[34] Zh.-H. Yang, "Necessary and sufficient conditions for Schur geometrical convexity of the four-parameter homogeneous means," Abstract and Applied Analysis, vol. 2010, Article ID 830163, 16 pages, 2010.

[35] Zh.-H. Yang, "Schur harmonic convexity of Gini means," International Mathematical Forum. Journal for Theory and Applications, vol. 6, no. 13-16, pp. 747-762, 2011.

[36] H. N. Shi, Theory of Majorization and Analytic Inequalities, Harbin Institute of Technology Press, Harbin, China, 2013.

[37] Z. Shan, Geometric Inequalities (in Chinese), Shanghai Education Press, Shanghai, China, 1980.

[38] D. S. Mitrinović, J. E. Pečarić, and V. Volenec, Recent Advances in Geometric Inequalities, vol. 28, Kluwer Academic Publishers, Dordrecht, The Netherlands, 1989.

[39] J. Z. Zhang and L. Yang, "A class of geometric inequalities concerning systems of mass points," Journal of China University of Science and Technology, vol. 11, no. 2, pp. 1-8, 1981.

[40] G. S. Leng, T. Y. Ma, and X. Z. Qian, "Inequalities for a simplex and an interior point," Geometriae Dedicata, vol. 85, no. 1-3, pp. 1-10, 2001.

[41] S. G. Yang, W. Wang, J. J. Pan, and D. Qian, "The ndimensional Pedoe inequality in hyperbolic space with applications," Advances in Mathematics, vol. 41, no. 3, pp. 347-355, 2012.

[42] J. Z. Xiao and X. H. Zhu, "Schur convex functions and inequalities of $n$-simplices," Applied Mathematics. A Journal of Chinese Universities A, vol. 16, no. 4, pp. 428-434, 2001. 


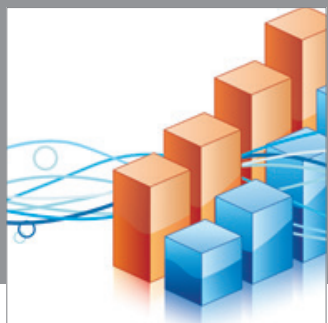

Advances in

Operations Research

mansans

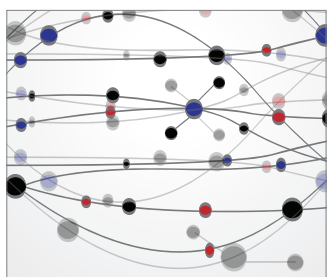

The Scientific World Journal
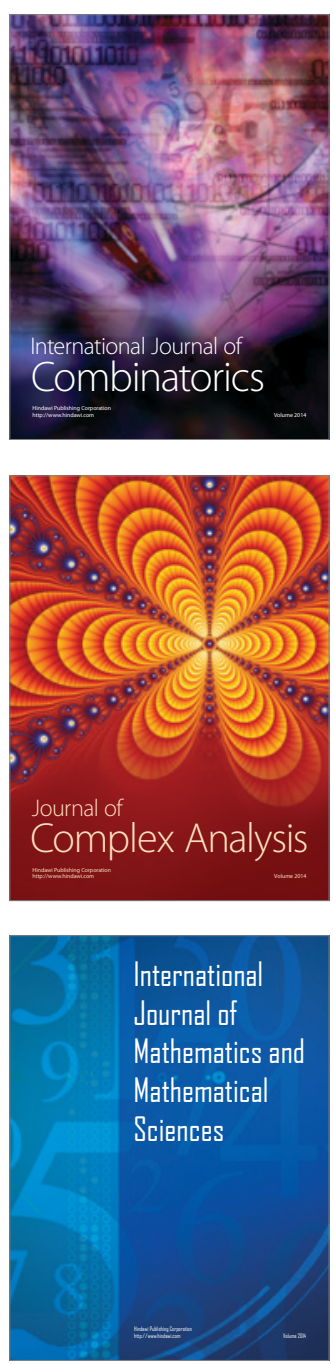
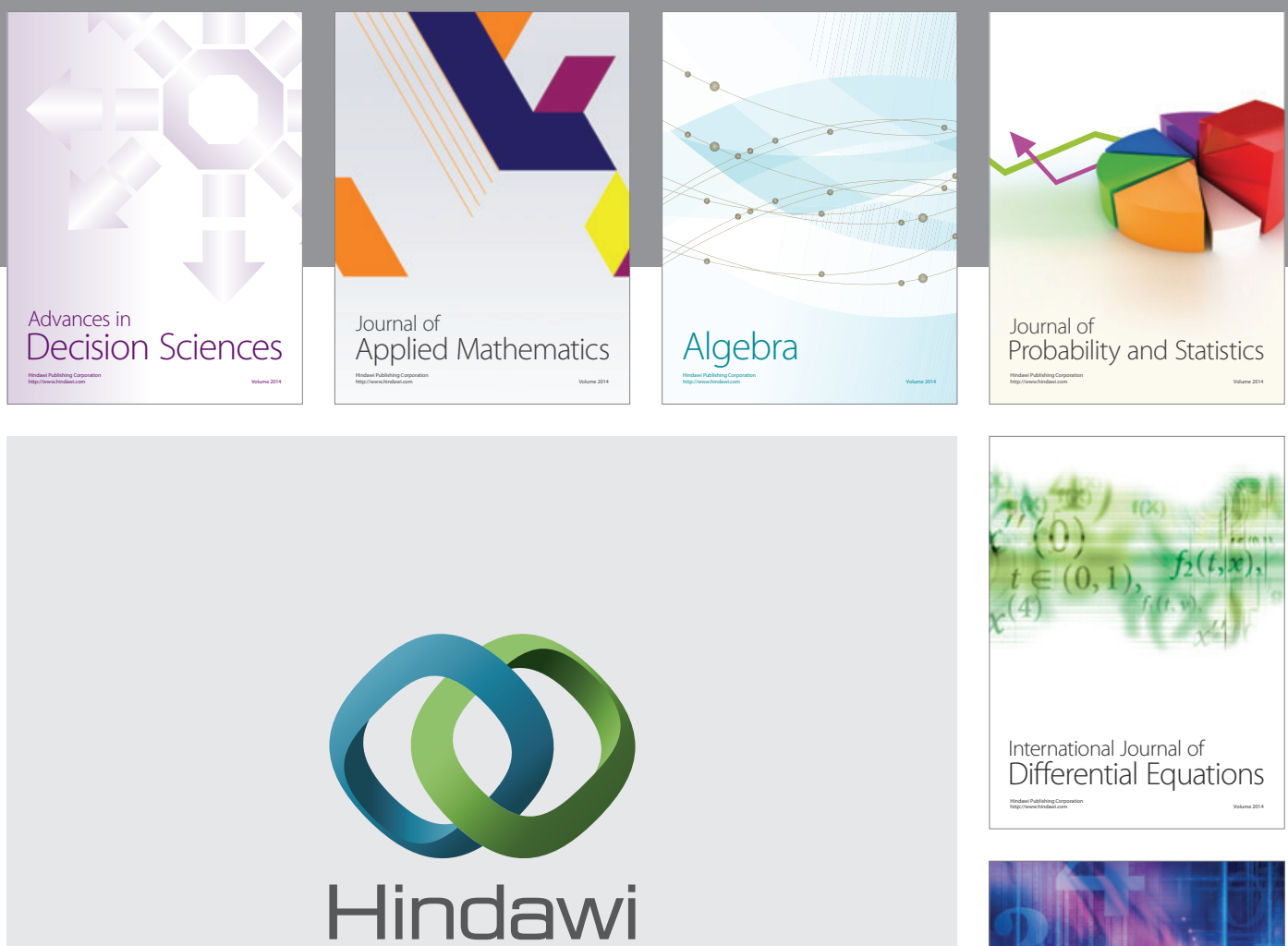

Submit your manuscripts at http://www.hindawi.com
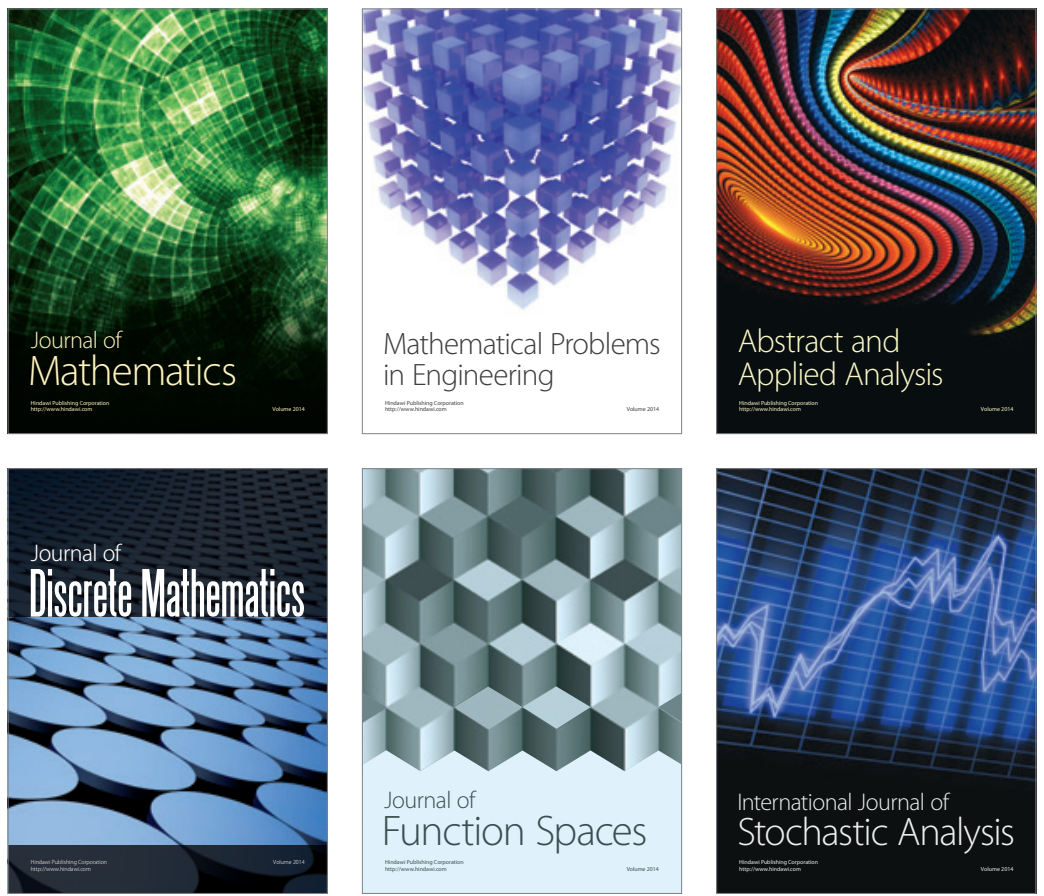

Journal of

Function Spaces

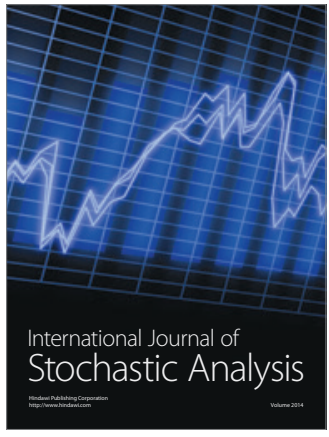

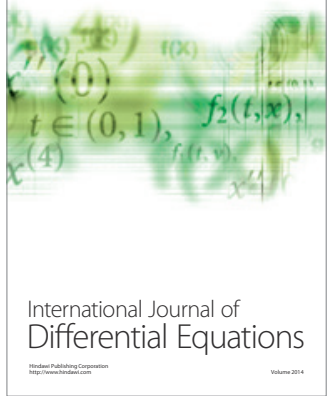
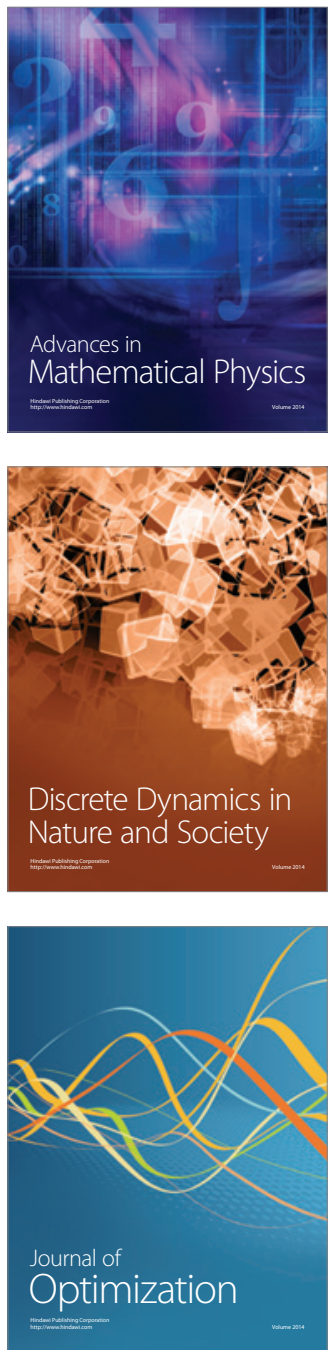